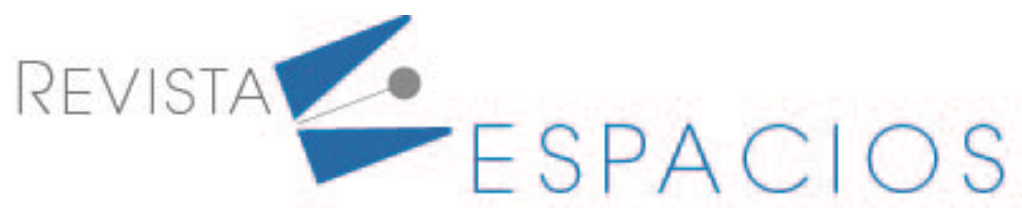

\title{
Diseño de aplicativo identificador de mascotas haciendo uso de códigos QR
}

\section{Pet identifier application design using QR codes}

\author{
SIMANCA, Fredys A. ${ }^{1}$ \\ GAMA, Oscar M. ${ }^{2}$ \\ CRIOLLO, Juan D. ${ }^{3}$ \\ PAEZ, Jaime $A^{4}$ \\ CORTES, Jairo A. ${ }^{5}$
}

Resumen. El presente artículo describe el diseño de un collar que porta un código QR para la identificación de las mascotas por medio de una aplicación de software. Se almacena el registro de sus datos y toda la información que los dueños consideren relevante. Este desarrollo permitió que un sector de estudiantes realizase un registro exitoso de sus mascotas en el aplicativo, demostrando un correcto funcionamiento.

Palabras clave: código qr, identificación de mascotas, aplicación móvil

\begin{abstract}
This article describes the design of a collar that carries a QR code for the identification of pets by means of a software application. The record of your data and all the information that the owners consider relevant is stored. This development allowed a sector of students to make a successful registration of their pets in the application, demonstrating correct operation.

key words: qr code, pet id, mobile app
\end{abstract}

\section{Introducción}

Actualmente el código QR se utiliza para diferentes usos, como: Acceso a información de una página Web, a una red social, recursos multimedia y mapas interactivos de ubicación, entre otros (Chang et al., 2012) (Alvarez Loaiza et al., 2019).

Consiste en un código de barra en dos dimensiones, que, en comparación a otros códigos, incluyendo los clásicos de una dimensión pueden almacenar más información. En la actualidad es muy sencillo generar estos códigos por medio de internet y pueden ser reconocido fácilmente a través de una cámara de un teléfono móvil $3 G$.

Sus aplicaciones pueden ser varias; tanto en la difusión de publicidad de productos, como en identificación de tiquetes electrónicos, también puede ser utilizado en temas de salud. En la actualidad son principalmente

\footnotetext{
${ }^{1}$ Profesor Investigador. Ingeniería de Sistemas. Universidad Libre. fredysa.simancah@unilibre.edu.co

2 Estudiante. Ingeniería de Sistemas .Universidad Libre. oscarm-gamag@unilibre.edu.co

3 Estudiante. Ingeniería de Sistemas . Universidad Libre. juand-criollop@unilibre.edu.co

${ }^{4}$ Profesor Investigador. Ingeniería de Sistemas. Universidad Cooperativa de Colombia. Jaime.paez@campusucc.edu.co

${ }^{5}$ Profesor Investigador. Ingeniería de Sistemas. Universidad Cooperativa de Colombia, jairo.cortes@campusucc.edu.co
} 
utilizados para facilitar identificación de información importante sin necesidad de tener una cantidad de papeles que contenga datos a los cuales se quiere acceder (UNITAG, 2020).

Otra de las aplicaciones que se encontraron de los códigos QR es la que realiza la fundación "Protege tu salud", esta se encarga de proteger a diabéticos ante situaciones de emergencia; las personas que sufren de diabetes cargan un carné el cual contiene un código QR que almacena los productos que deben ser suministrados en caso de emergencia para que se pueda garantizar su seguridad - (Infodiabético, 2020).

En el tema de salud el uso de códigos QR se ha utilizado para distribuir información de manera efectiva y simple (Lombardo et al., f2012). Por ejemplo, Taiwán viene utilizando el código QR de manera exitosa y se ha evaluado su uso para transmitir la información y prescripción de un paciente desde el hospital a la farmacia (Lin et al., (2012). Así mismo, se han descrito otras experiencias, en la educación de pacientes y para facilitar el trabajo de imagenología médica (Ritter et al., 2012) Contreras et al., 2019).

Otro de los usos identificados fue el que se empezó a dar en la declaración de la renta desde el año 2017 en España (El Periódico, 2018), la APP de Hacienda, que fue en la que se realizó la declaración de renta en ese año, permite a los ciudadanos acceder a nuevos servicios para los que se requiere identificación, a través del código QR de los documentos recibidos en Hacienda.

Básicamente todas estas aplicaciones de los códigos QR mencionadas se basan en la recolección de información, y el punto diferenciador se encuentra en la forma de presentarla, ya que para que una persona quiera acceder a ella, solo tendrá que escanear el código QR desde su móvil e inmediatamente tendrá acceso a lo que este contiene (Radicelli et al., 2019).

Los códigos QR pueden brindar una solución en este caso muy efectiva implementándolos como collares que utilízan las mascotas, almacenando en ellos la información, como por ejemplo, Nombre, Edad, Nombre de los o el dueño, número de contacto, dirección o ubicación por medio de Google Maps de su residencia; en el caso de que esta mascota sufra de alguna enfermedad o necesite ser tratado con un medicamento se acceda a la información necesaria en este código QR, y de esta forma poder identificarla en caso de cualquier eventualidad.

El diseño de la solución planteada consta de un desarrollo móvil con una interfaz amigable y fácil de usar para una mejor experiencia de los usuarios de la aplicación. Actualmente hay celulares móviles que ya traen preinstalados lectores de códigos QR como por ejemplo Apple que en su nueva versión de sistema operativo IOS 11 tiene una herramienta implementada en sus opciones de cámara que permite leer códigos QR sin necesidad de aplicaciones externa (iPadizate, 2017).

Se pretende entonces desarrollar una herramienta que sea útil en el momento dado de que una persona encuentre una mascota extraviada o que allá sufrido un accidente y por medio del código QR pueda identificarla y ponerse en contacto con el dueño del animal.

La funcionalidad de la aplicación dependerá mucho de la comunidad ya que es necesario que los dueños de las mascotas hagan el registro de ellas en la plataforma y que cuando una persona encuentre un animal realice la identificación y se ponga en contacto con los dueños.

Mediante este proyecto se quiere presentar el uso de esta potente herramienta para identificación, siendo más puntuales, llevarlo a la identificación de mascotas para diversos usos primordiales ya que hoy en día ocupan un lugar en la familia muy importante, y se convierten en parte indispensable de ella, sus dueños de esta manera buscan que ellas sean identificadas en la sociedad y del mismo modo brindarles protección estando en todo momento pendiente de ellas. 
Se espera que el buen uso de esta herramienta contribuya a bajar el número de mascotas extraviadas empezado en la ciudad de Bogotá, y los dueños de estas se sientan más tranquilos sabiendo que existe una comunidad que está al pendiente de las mascotas que hagan parte de esta plataforma.

\section{Metodología}

Se desarrolló una metodología por fases, en la primera se realizó una investigación en la Universidad Libre seccional Bogotá, sede Bosque Popular, la cual buscaba identificar cuantos estudiantes tendrían mascotas y estarían dispuestos a usar la solución diseñada.

Inicialmente los usuarios fueron estudiantes de la Universidad Libre ya que esta población abarca una mayor área de cobertura de la ciudad y así facilitar el encuentro de mascotas.

En esta fase se realizó una encuesta a los estudiantes buscando identificar qué tipo de mascota tenía esta población y cuantos estarían dispuestos a realizar un registro en el aplicativo, la entrevista nos arrojó los siguientes resultados (Ver figura 1).

Figura 1

Cantidad de mascotas en la Universidad Libre

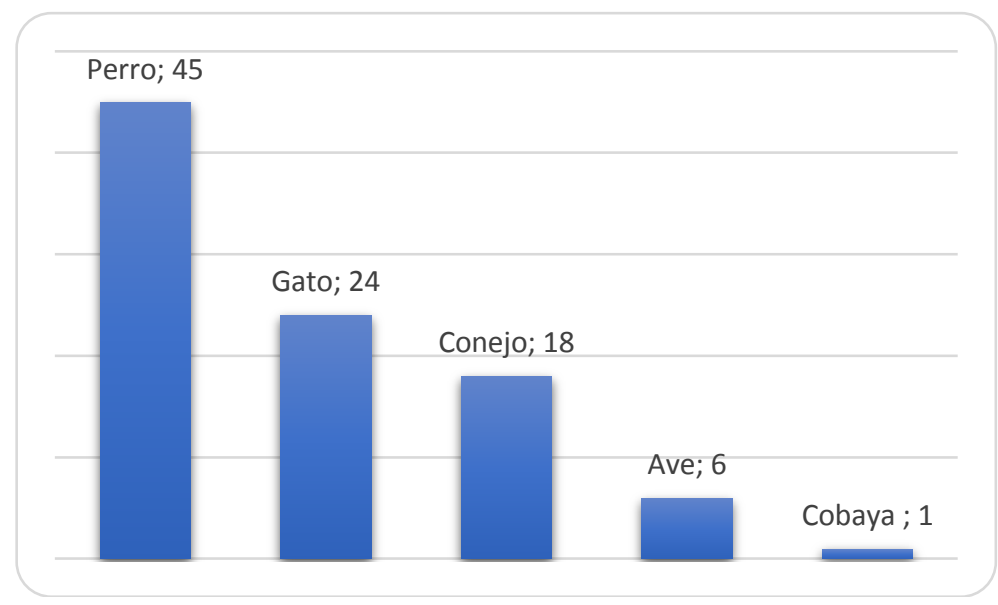

Fuente: Elaboración propia

De las 102 personas encuestadas 94 tienen en su casa una mascota y estarían dispuestos a registrarse en el aplicativo.

El lenguaje en el que se programó la aplicación fue JavaScript ya que esta herramienta brinda una compatibilidad y permite crear páginas dinámicas y llamativas, dando como resultado un uso sencillo para el usuario (Next_U, 2020). Se diseñó una página web para poder registrar y llevar un control de las mascotas ingresadas a la plataforma, cabe aclarar que desde este dispositivo no se tendrá la función de escanear códigos QR (es netamente informativo). La aplicación será completamente gratuita en sus funcionalidades básicas como lo es el registro, el ingreso de datos y escáner códigos QR, la cual es una acción que se puede realizar con o sin la aplicación.

En la segunda fase de Dimensionamiento se realizaron los diseños de las interfaces. En esta fase se permitió identificar que el motor de la base de datos a trabajar fuera ORACLE 12c. Se hizo uso de la metodología de prototipos en el desarrollo para poder en el futuro implementar cambios que puedan surgir. 
En esta fase de diseño de interfaz se tuvo presente los requerimientos y el objetivo fundamental de la aplicación que era colaborar a los dueños de macotas a identificarlas en caso de que estas se extravíen, con ayuda de los demás usuarios que utilicen la aplicación.

\section{Resultados}

El diseño del software para la identificación de mascotas haciendo uso de un código QR condujo al desarrollo de una aplicación estructurada en los siguientes módulos: módulo de autenticación, módulo de generación de código y datos de la mascota, módulo de escáner de qr.

Módulo 1 Autenticación. Este módulo permite el registro y posterior autenticación de los usuarios se piden datos básicos como: correo, contraseña, un número telefónico y su dirección (Ver Figura 2)

Figura 2

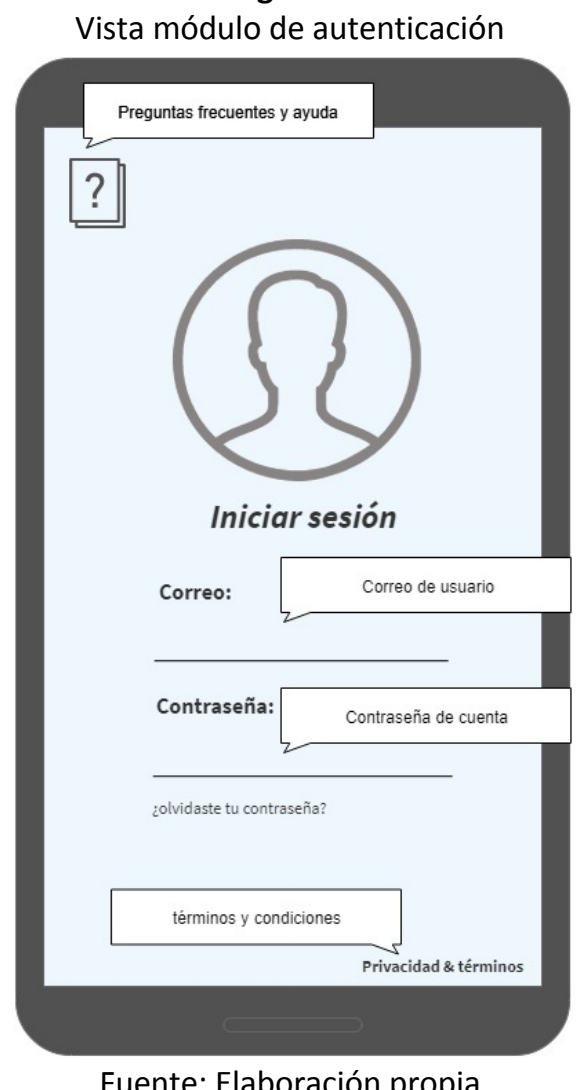

Fuente: Elaboración propia

Módulo 2. Registro de mascotas. En la Figura 3 se muestra el módulo de los datos de mascota, allí se realizar el registro de la mascota ingresando datos básicos como: Tipo de mascota, nombre, edad, raza, características, entre otros. 
Figura 3

Vista registro datos de la Mascota

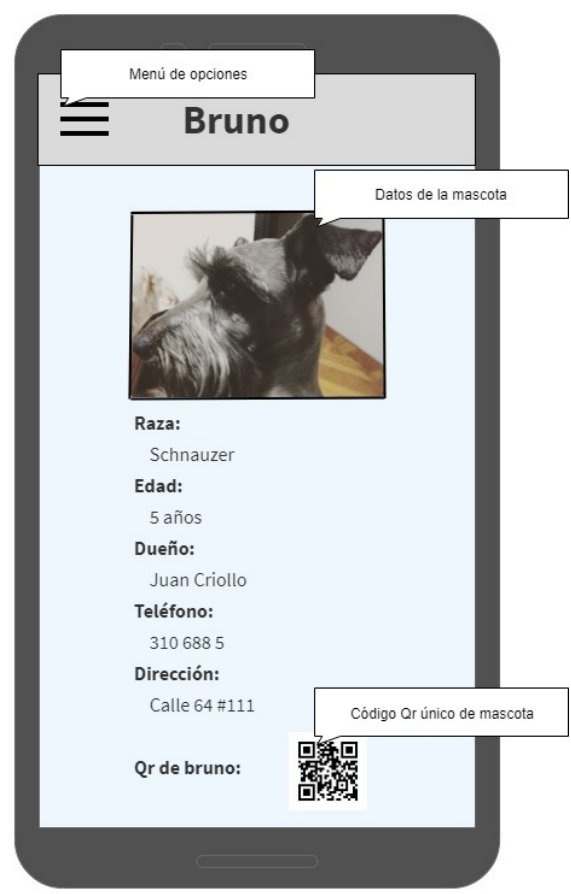

Fuente: Elaboración propia

Módulo 3. Generación de código QR. En este módulo se genera código QR único de la mascota se muestra en la Figura 3 que posteriormente será adherido al collar de la mascota, al dar doble clic sobre este se descargará del celular un PDF del código para ser descargado.

Luego de la generación el propietario podrá adherir el código QR el collar de la mascota como se muestra en la Figura 4.

Figura 4

Prototipo de collar

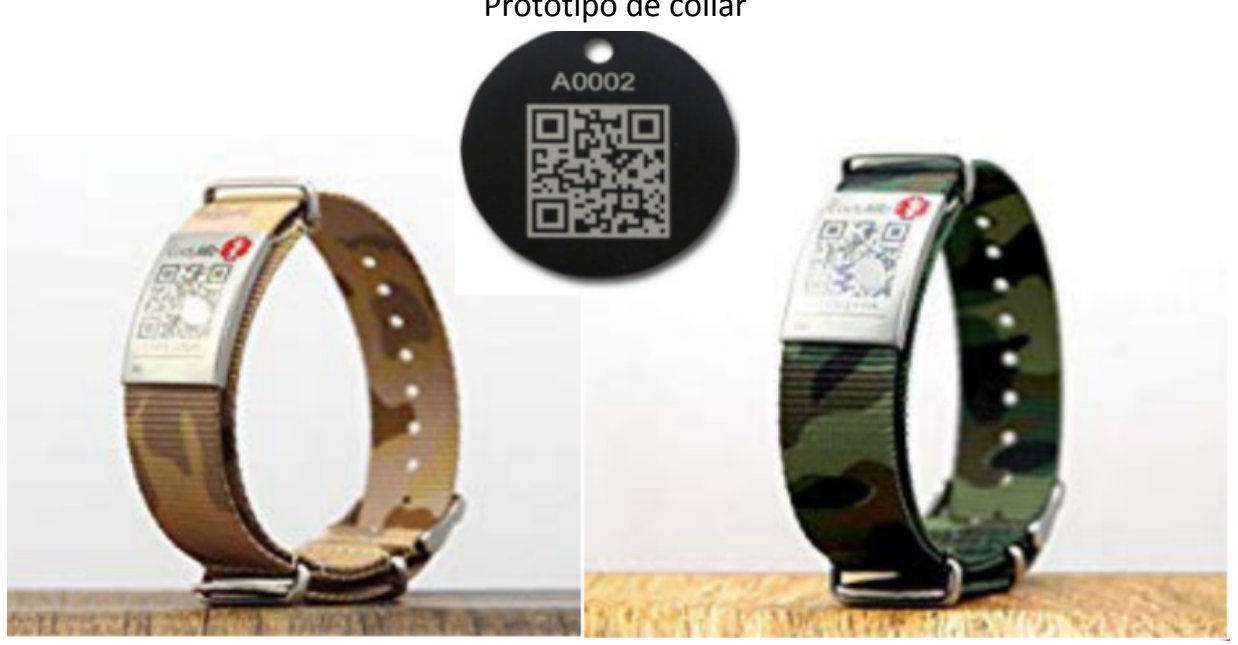

Fuente: Elaboración propia

En la Figura 4, se tiene la opción para que el usuario pueda escanear un código QR de la mascota en caso de que se encuentre con una y quiera acceder a los datos, esta misma autenticación se puede realizar desde cualquier celular siempre y cuando cuente con la herramienta para esta función, no es necesario realizar el escaneo del código estando dentro de la aplicación (Ver figura 5). 
Figura 5

Escaner de códigos QR

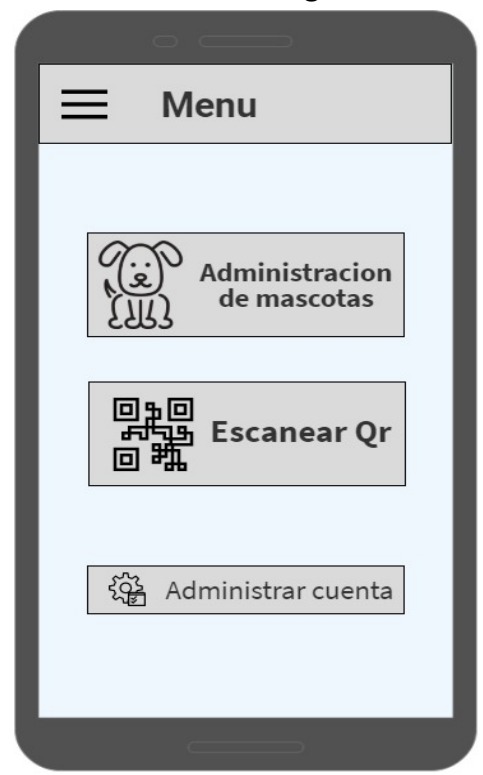

Fuente: Elaboración propia

Para escanear el código simplemente se accede a la acción del aplicativo o se activa la cámara del celular. Escaneando el código se podrá tener acceso a la información de la mascota como se puede apreciar en la Figura 6.

Figura 6

Escaner de códigos QR

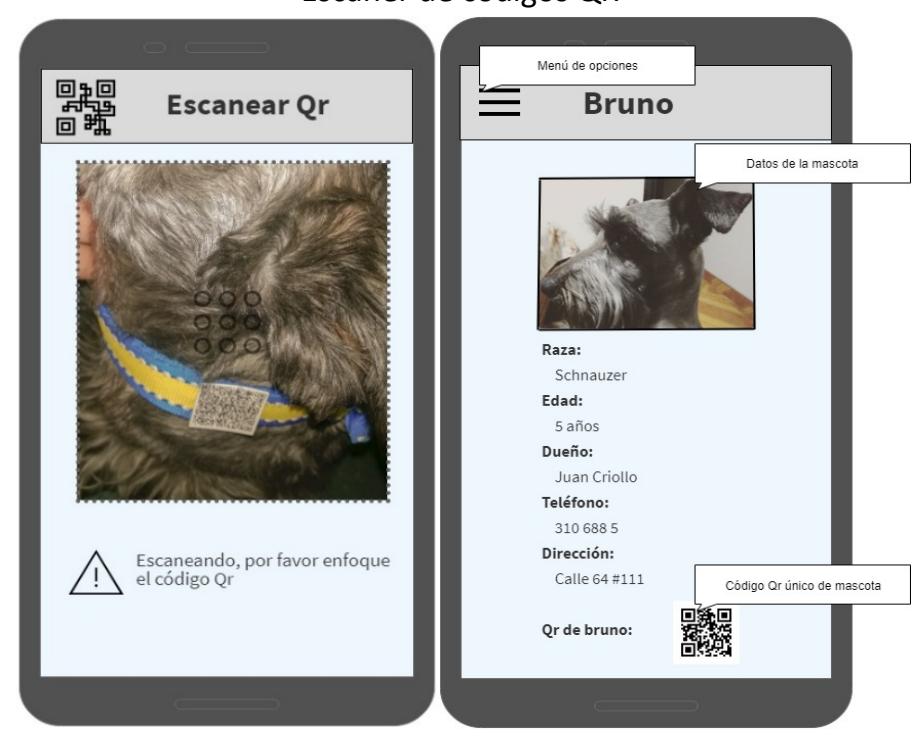

Fuente: Elaboración propia

\subsection{Validación de la herramienta}

La herramienta la utilizaron 45 personas en el rango de tiempo noviembre 2018 - abril 2019, realizando registro exitoso de los datos de sus mascotas. En el primer del año 2019 se reportaron 5 mascotas recuperadas con ayuda de esta aplicativo. 


\section{Discusión}

Si bien es cierto que hoy en día existen aplicaciones tales como el chip localizador, pero el problema que tiene este método es que cuenta con un rango limitado para poder ser localizado y si no se cuenta con la herramienta especial que permita leer este chip al final no podrá ser identificada. Aparte de ello este se considera como un método algo invasivo para la mascota.

El desarrollo de esta aplicación ofrece la posibilidad de ser usada en cualquier lugar y en todo momento ya que con dispositivo móvil se puede escanear los códigos que tengan las mascotas. También como dueño se puede editar la información que se desea que aparezca en el código QR y hacer el registro de todas las macotas que posea, esto le garantizara que los datos de estas se encuentran en un servidor y podrán ser visto por personas que escaneen dicho código, esto podrá facilitar acciones de búsqueda de la mascota cuando sea necesario.

Es una aplicación gratuita la cual le permitirá al usuario realizar un ilimitado registro de mascotas e imprimir sus códigos correspondientes, esto hará que muchos usuarios la utilicen sin problema alguno.

Un factor a tener en cuenta es que el hecho de encontrar una mascota, identificarla y devolverla es más una decisión de ética personal, como tal no existe un control que obligue a la persona a devolverla.

\section{Conclusiones}

Gracias al desarrollo de este tipo de software que implementa lectura de códigos QR se puede obtener un ingreso más ágil a vínculos que contienen información de interés y al ser aplicado a la identificación de mascotas se contribuyó al acceso de datos estas de una forma más eficaz dado caso de llegar a ser necesitados.

El software busca almacenar toda la información que los dueños de mascotas quieran publicar, se puede igualmente publicar información médica de la mascota en caso de que esta lleve un trato especial o simplemente cambie de veterinario.

Gracias a los resultados arrojados en las encuestas donde se evidencia un gran número de personas que cuenta con mascota y a su vez les agradaría utilizar esta aplicación se concluye que el plan piloto de la aplicación se desarrolló principalmente en la Universidad Libre seccional Bogotá sede bosque popular ya que los estudiantes se encuentran bien distribuidos en la capital, esto ofrecerá que la aplicación se recomiende y el número de usuarios crezca constantemente y bien distribuido en la ciudad para que de esta manera también ayude a la búsqueda de mascotas en un área grande en caso de que estas se pierdan. El éxito de esta aplicación se garantizará si crece en gran medida sus usuarios por lo tanto esto es lo que se quiere logar; una aplicación amigable con los usuarios y que garantice una excelente funcionalidad.

\section{Referencias bibliográficas}

\section{Bibliografía}

Alvarez Loaiza, P., Vite Valverde, F., Yánez Moretta, P., Burneo Villegas, C., \& Jumbo Ramos, S. (2019). Modelo de aplicación del código QR (Quick Response Code) en actividades de gestión y educación ambientales en un jardín botánico del Neotrópico (Ecuador). Espacios, 40(16), 23-43.

Chang, L., Linchun, S., Xiaolan, X., Huan, L., Hang, X., Dong, L., Shilin, C. (2012). DNA Barcode Goes TwoDimensions: DNA QR Code Web Server. PLos One, 7(5), 1-7.

Contreras, M., Barrios, E., \& Rodriguez, J. (2019). Reconocimiento y rastreo de imágenes en aplicaciones de realidad aumentada. Espacios, 40(34), 7-22. 
El Periódico. (2018). Así funciona la app de la declaración de la renta 2017. El Periódico. Retrieved from https://www.elperiodico.com/es/economia/20180315/app-declaracion-renta-2017-6691832

Infodiabético. (2020). https://www.infodiabetico.com/. Retrieved from https://www.infodiabetico.com/: https://www.infodiabetico.com/index.php/productos/3014-protege-tu-salud-te-ayuda-a-protegerte-antesituaciones-de-emergencia

iPadizate. (2017). https://www.ipadizate.es/. Retrieved from https://www.ipadizate.es/: https://www.ipadizate.es/2017/07/29/codigos-qr-con-camara-ios-11

Lin, C., Tsai, F., Tsai, W., Wen, H., \& Hu, M. (2012). The feasibility of QR-code prescription in Taiwan. Clin Pharm Ther, 37(6), 643-646.

Lombardo, N., Morrow, A., \& Le Ber, J. (2012). Rethinking Mobile Delivery: Using Quick Response Codes to Access Information at the Point of Need. Medical Reference Services Quarterly, 31(1), 14-24.

Next_U. (2020, 10 10). https://www.nextu.com/. Retrieved from https://www.nextu.com/: https://www.nextu.com/blog/conoce-las-ventajas-y-desventajas-de-javascript/

Radicelli, C., Pomboza, M., Samaniego, N., Villacrpes, E., \& Pomboza, C. (2019). Códigos de respuesta rápida: su utilización en el aspecto académico - investigativo de la Universidad Nacional de Chimborazo. Ecuador. Espacios, 40(41), 27-36.

Ritter, F., Thorsten Wenzel, M., Al Issawi, J., Benten, S., \& Schillin, K. (2012). Combining Mobile Devices and Workstations for the Reading of Medical Images. i-Com, 12(1).

UNITAG. (2020). https://www.unitag.io/. Retrieved from https://www.unitag.io/: https://www.unitag.io/es/qrcode/what-is-a-qrcode 